\title{
Polarization-Free Generators and the S-Matrix
}

\author{
Hans--Jürgen Borchers ${ }^{a}$, Detlev Buchholz ${ }^{a}$ and Bert Schroer $^{b}$ \\ ${ }^{a}$ Institut für Theoretische Physik, Universität Göttingen \\ Bunsenstraße 9, 37073 Göttingen, Germany \\ ${ }^{b}$ Fachbereich Physik, Freie Universität Berlin \\ Arnimallee 14, 14195 Berlin, Germany \\ Dedicated to the memory of Harry Lehmann
}

\begin{abstract}
Polarization-free generators, i.e. "interacting" Heisenberg operators which are localized in wedge-shaped regions of Minkowski space and generate single particle states from the vacuum, are a novel tool in the analysis and synthesis of two-dimensional integrable quantum field theories. In the present article, the status of these generators is analyzed in a general setting. It is shown that such operators exist in any theory and in any number of spacetime dimensions. But in more than two dimensions they have rather delicate domain properties in the presence of interaction. If, for example, they are defined and temperate on a translation-invariant, dense domain, then the underlying theory yields only trivial scattering. In two-dimensional theories, these domain properties are consistent with non-trivial interaction, but they exclude particle production. Thus the range of applications of polarization-free generators seems to be limited to the realm of two-dimensional theories.
\end{abstract}

\section{Introduction}

Local quantum field theory provides the adequate setting for elementary particle physics. It allows one to express in mathematical terms the basic features of relativistic quantum physics, such as Einstein causality, Poincaré covariance and the Nahwirkungs-principle, which are encoded in the local field equations and commutation relations. The physical interpretation of the theory relies on asymptotic notions, however, based on the particle concept.

The way from the local fields to the asymptotic particle interpretation was paved in the seminal work of Lehmann, Symanzik and Zimmermann [1] who invented a consistent collision theory and established reduction formulas for the 
computation of scattering matrix elements. Little is known about the opposite road, however, i.e. the reconstruction of a local theory from a given scattering matrix. This problem, sometimes called form-factor program [2], is for example of importance in the construction of integrable field-theoretic models, cf. [3] for some interesting progress in this respect.

It was recently noticed [4] that certain interacting theories in two spacetime dimensions admit an important intermediate step in this program. Namely, there exist semi-local polarization-free generators, which are localized in wedge-shaped regions of Minkowski space and generate from the vacuum single particle states, similarly to free fields. Important features of the theory, such as the crossing symmetry of the scattering matrix, are encoded in simple analyticity properties of the correlation functions of these operators (KMS-condition). Moreover, their algebraic properties can directly be expressed in terms of the elastic scattering amplitudes. This interesting observation warrants a more systematic investigation of polarization-free generators in the general setting of local quantum field theory.

The present article is devoted to such a study. We will show that there exist polarization-free generators in any local theory with a non-trivial particle spectrum, irrespectively of the number of spacetime dimensions. It turns out, however, that these operators are generically unbounded and their domains of definition exhibit some delicate features if there is interaction. If, for example, the polarization-free generators are defined on a translation invariant domain and the norms of their respective images stay polynomially bounded for large translations, then the elastic scattering amplitudes inevitably vanish in more than two spacetime dimensions. In two-dimensional theories, these domain properties are consistent with interaction, but they exclude particle production. Thus the only non-trivial theories in which such temperate families of polarization-free generators can be defined seem to be models of the type studied in [四].

The upshot of our investigation is the insight that polarization-free generators always exist. But in view of their subtle domain properties, they are not accessible to Fourier analysis in most cases of physical interest. They are therefore not suitable for a general analysis and synthesis of collision states and do not provide the desired universal link between the scattering matrix and the local interacting fields. Some further aspects of our results are mentioned in the conclusions.

\section{Existence of polarization-free generators}

Explicit non-trivial examples of polarization-free generators were invented in some two-dimensional relativistic quantum field theories [4]. But their generic features can be stated more clearly in the general framework of local quantum physics [5]. For the convenience of the reader who is not familiar with this setting, we briefly recall in the following the relevant notions and explain our notation.

We assume that we are given a local, relativistic quantum field theory in $d$ dimensional Minkowski space $\mathbb{R}^{d}$. But instead of dealing with the unbounded field 
operators, we proceed to their local bounded functions [6]. Each of the resulting bounded operators is associated to some region $\mathcal{O} \subset \mathbb{R}^{d}$, fixed by support of the test functions involved in the smearing of the field operators. We say that these operators are localized in $\mathcal{O}$, for short. The collection of all operators localized in a particular region $\mathcal{O}$ generates a ${ }^{*}$-algebra $\mathcal{A}(\mathcal{O})$ on the underlying physical Hilbert space $\mathcal{H}$ which is closed in the weak operator topology. (It is thus a von Neumann algebra.) The family of these local algebras inherits from the underlying quantum field theory the following fundamental properties:

1. (Locality) The assignment

$$
\mathcal{O} \rightarrow \mathcal{A}(\mathcal{O})
$$

defines a net over Minkowski space, i.e. an inclusion preserving mapping. The net complies with the principle of locality, that is operators affiliated with spacelike separated regions commute.

Besides these "local algebras", we also consider algebras $\mathcal{A}(\mathcal{W})$ associated to wedge-shaped regions $\mathcal{W}$ of the form (given in proper coordinates)

$$
\mathcal{W}_{1}=\left\{x \in \mathbb{R}^{d}: x_{1} \geq\left|x_{0}\right|, x_{2}, \ldots x_{d-1} \text { arbitrary }\right\}
$$

as well as their Poincaré transforms. They are the smallest von Neumann algebras containing all local algebras $\mathcal{A}(\mathcal{O})$ with $\mathcal{O} \subset \mathcal{W}$. Because of locality, the algebra $\mathcal{A}\left(\mathcal{W}^{\prime}\right)$ associated to the spacelike complement $\mathcal{W}^{\prime}$ of $\mathcal{W}$ commutes with $\mathcal{A}(\mathcal{W})$, $\mathcal{A}\left(\mathcal{W}^{\prime}\right) \subset \mathcal{A}(\mathcal{W})^{\prime}$

2. (Covariance) The group of spacetime translations $\mathbb{R}^{d}$ acts on $\mathcal{H}$ by a continuous unitary representation $U$ which induces automorphisms of the net. Thus for any translation $x \in \mathbb{R}^{d}$ and region $\mathcal{O} \subset \mathbb{R}^{d}$

$$
U(x) \mathcal{A}(\mathcal{O}) U(x)^{-1}=\mathcal{A}(\mathcal{O}+x)
$$

in an obvious notation.

3. (Spectrum) The joint spectrum of the generators $P$ of $U$ (the physical energymomentum spectrum) is contained in the closed forward lightcone,

$$
\operatorname{sp} P \subset\left\{p \in \mathbb{R}^{d}: p_{0} \geq|\boldsymbol{p}|\right\} .
$$

Moreover, there is a unit vector $\Omega \in \mathcal{H}$, unique up to a phase, which is invariant under the action of $U$ and cyclic for the local algebras $\mathcal{A}(\mathcal{O})$ (Reeh-Schliederproperty). This vector describes the vacuum state.

Because of this familiar form of the energy-momentum spectrum, the mass operator

$$
M=\left(P_{0}^{2}-\boldsymbol{P}^{2}\right)^{1 / 2}
$$

is positive selfadjoint with spectral resolution $E(\cdot)$. If there are particles of mass $m$ in the theory, the spectral projection $E_{m}=E(\{m\})$ is different from zero. It is our aim to show that there exist operators which are localized in wedge regions and generate from the vacuum single particle states with mass $m$.

The formal characterisation of such operators is given in the subsequent definition. We recall in this context that a closed operator is said to be affiliated 
with a von Neumann algebra $\mathcal{M}$ if it commutes on its domain with all elements of the commutant $\mathcal{M}^{\prime}$ of $\mathcal{M}$. Its adjoint is then also affiliated with $\mathcal{M}$.

Definition: A closed operator $G$ is called polarization-free generator of mass $m$ if (a) it is affiliated with a wedge algebra $\mathcal{A}(\mathcal{W})$, (b) $\Omega$ is contained in the domains of $G$ and $G^{*}$, and (c) $G \Omega, G^{*} \Omega$ are elements of $E_{m} \mathcal{H}$.

For the proof that polarization-free generators exist in any theory, we make use of Tomita-Takesaki-Theory [7]. We begin by recalling some basic facts from this theory for the case at hand. Since $\Omega$ is cyclic and separating for the wedgealgebras $\mathcal{A}(\mathcal{W})$ by the Reeh-Schlieder property and locality, one can consistently define the Tomita conjugations $S_{\mathcal{W}}$, setting

$$
S_{\mathcal{W}} A \Omega=A^{*} \Omega, \quad A \in \mathcal{A}(\mathcal{W})
$$

These operators are closable anti-linear involutions. Their closures, which we denote by the same symbol, have the polar decomposition

$$
S_{\mathcal{W}}=J_{\mathcal{W}} \Delta_{\mathcal{W}}^{1 / 2}
$$

Here $J_{\mathcal{W}}$ is an anti-unitary operator, the modular conjugation, and $\Delta_{\mathcal{W}}$, the modular operator, is strictly positive and selfadjoint. The following well-known fact is of fundamental importance in the present context. We therefore sketch its proof.

Lemma 2.1 Let $\Phi$ be any vector in the domain of $S_{\mathcal{W}}$. There exists a closed operator $F$ which (a) is affiliated with $\mathcal{A}(\mathcal{W})$, (b) has, together with its adjoint $F^{*}$, the vector $\Omega$ in its domain and (c) satisfies

$$
F \Omega=\Phi, \quad F^{*} \Omega=S_{\mathcal{W}} \Phi
$$

Proof: Since the set of vectors $\mathcal{A}(\mathcal{W}) \Omega$ is a core fore $S_{\mathcal{W}}$ and $S_{\mathcal{W}}$ is closed, there is a sequence $F_{n} \in \mathcal{A}(\mathcal{W})$ such that $F_{n} \Omega \rightarrow \Phi$ and $S_{\mathcal{W}} F_{n} \Omega \rightarrow S_{\mathcal{W}} \Phi$, strongly. Thus if $A^{\prime} \in \mathcal{A}(\mathcal{W})^{\prime}$, one also has $F_{n} A^{\prime} \Omega=A^{\prime} F_{n} \Omega \rightarrow A^{\prime} \Phi$ and $F_{n}^{*} A^{\prime} \Omega=A^{\prime} F_{n}^{*} \Omega \rightarrow$ $A^{\prime} S_{\mathcal{W}} \Phi$. So the operator $F$, given by

$$
F A^{\prime} \Omega=\lim _{n \rightarrow \infty} F_{n} A^{\prime} \Omega=A^{\prime} \Phi, \quad A^{\prime} \in \mathcal{A}(\mathcal{W})^{\prime},
$$

is well defined. Its adjoint $F^{*}$ also has the dense set of vectors $\mathcal{A}(\mathcal{W})^{\prime} \Omega$ in its domain and

$$
F^{*} A^{\prime} \Omega=\lim _{n \rightarrow \infty} F_{n}^{*} A^{\prime} \Omega=A^{\prime} S_{\mathcal{W}} \Phi, \quad A^{\prime} \in \mathcal{A}(\mathcal{W})^{\prime} .
$$

This shows that $F$ is closable (we use the symbol $F$ also for its closure), and it establishes part (b) and (c) of the statement since $1 \in \mathcal{A}(\mathcal{W})^{\prime}$. For part (a) one makes use of the fact that for any vector $\Psi^{*}$ in the domain of $F^{*}$ and $A^{\prime}, B^{\prime} \in \mathcal{A}(\mathcal{W})^{\prime}$

$$
\left(A^{\prime} \Psi^{*}, F B^{\prime} \Omega\right)=\left(\Psi^{*}, A^{*} B^{\prime} \Phi\right)=\left(\Psi^{*}, F A^{*} B^{\prime} \Omega\right)=\left(A^{\prime} F^{*} \Psi^{*}, B^{\prime} \Omega\right) .
$$


Hence $\left|\left(A^{\prime} \Psi^{*}, F B^{\prime} \Omega\right)\right| \leq$ const $\cdot\left\|B^{\prime} \Omega\right\|, B^{\prime} \in \mathcal{A}(\mathcal{W})^{\prime}$. So $A^{\prime} \Psi^{*}$ lies in the domain of $F^{*}$ and $F^{*} A^{\prime} \Psi^{*}=A^{\prime} F^{*} \Psi^{*}, A^{\prime} \in \mathcal{A}(\mathcal{W})^{\prime}$. An analogous statement holds for $F^{* *}=F$, so the proof of the lemma is complete.

In view of this lemma, it suffices for the proof of the existence of polarizationfree generators to exhibit non-zero vectors $\Phi_{1} \in E_{m} \mathcal{H}$ in the domain of $S_{\mathcal{W}}$ such that also $S_{\mathcal{W}} \Phi_{1} \in E_{m} \mathcal{H}$. To accomplish this, we have to take a closer look at the modular operators and conjugations. Fortunately, we have sufficiently concrete information about these objects in the present general setting.

Since $\Delta_{\mathcal{W}}$ is strictly positive, we can proceed to the corresponding unitary group $\Delta_{\mathcal{W}}^{i s}, s \in \mathbb{R}$, called modular group. It is an important consequence of the spectral properties of the generators of $U$ and covariance 8 that for $x \in \mathbb{R}^{d}$

$$
\Delta_{\mathcal{W}}^{i s} U(x) \Delta_{\mathcal{W}}^{-i s}=U(\Lambda(s) x) \quad \text { and } \quad J_{\mathcal{W}} U(x) J_{\mathcal{W}}^{-1}=U(\Pi x),
$$

where $\Lambda(s), s \in \mathbb{R}$, is (with some appropriate scaling of $s$ ) the one-parameter group of boosts leaving the wedge $\mathcal{W}$ invariant and $\Pi$ is the reflection about the edge of $\mathcal{W}$. If, for example, $\mathcal{W}_{1}$ is the wedge given in $(2.2), x_{ \pm} \in \mathbb{R} \cdot( \pm 1,1,0, \ldots 0)$ are any two lightlike tranlations in the characteristic planes forming the boundary of $\mathcal{W}_{1}$, and $x_{\perp}=\left(0,0, x_{2}, \ldots x_{d-1}\right)$ is any translation along the edge of $\mathcal{W}_{1}$, then

$$
\Lambda_{1}(s) x_{ \pm}=e^{ \pm 2 \pi s} x_{ \pm}, \Lambda_{1}(s) x_{\perp}=x_{\perp} \quad \text { and } \quad \Pi_{1} x_{ \pm}=-x_{ \pm}, \Pi_{1} x_{\perp}=x_{\perp} .
$$

Thus the modular groups and conjugations act on the translations $U$ like Lorentz transformations. As a matter of fact, these operators generate a representation of the proper Poincaré group in generic cases according to the Bisognano-Wichmann theorem [9]. But this more detailed information is not needed here.

Knowing that the modular groups and conjugations act on the generators $P$ of $U$ like Lorentz transformations, we conclude that the mass operator $M$, being invariant under Lorentz transformations of $P$, commutes both with $J_{\mathcal{W}}$ and $\Delta_{\mathcal{W}}^{i s}, s \in \mathbb{R}$. The same is true for the spectral projections $E_{m}$ of $M$, hence $S_{\mathcal{W}}=J_{\mathcal{W}} \Delta_{\mathcal{W}}^{1 / 2}$ commutes on its domain $\mathcal{D}\left(S_{\mathcal{W}}\right)$ with $E_{m}$. This implies that $E_{m} \mathcal{D}\left(S_{\mathcal{W}}\right)$ is a dense subspace of $E_{m} \mathcal{H}$ which is stable under the action of $S_{\mathcal{W}}$ since $S_{\mathcal{W}}{ }^{2}=1$, cf. relation (2.6). Applying the preceding lemma, we have thus established the existence of polarization-free generators.

Theorem 2.2 Given any $m$ in the discrete spectrum of the mass operator and any wedge $\mathcal{W}$, there exist polarization-free generators $G$ of mass $m$ which are affiliated with $\mathcal{A}(\mathcal{W})$. In fact, for any vector $\Phi_{1}$ in the dense subspace $E_{m} \mathcal{D}\left(S_{\mathcal{W}}\right)$ of $E_{m} \mathcal{H}$, there is a $G$ such that $G \Omega=\Phi_{1}$ and $G^{*} \Omega=S_{\mathcal{W}} \Phi_{1} \in E_{m} \mathcal{D}\left(S_{\mathcal{W}}\right)$.

The simplest example illustrating this existence theorem is free field theory. We briefly discuss it here in order to indicate a subtle point in applications of this abstract result. Let $\phi_{0}$ be the free massive scalar field acting on Fock space. It is well-known [10] that the field operators $\phi_{0}(f)$, smeared with real test functions $f$ with compact support in some $\mathcal{O} \subset \mathbb{R}^{d}$, are essentially self-adjoint on the domain $\mathcal{D}_{0}$ consisting of all vectors with a finite particle number. They generate, 
by their spectral resolutions, the local algebras $\mathcal{A}_{0}(\mathcal{O})$ and are thus affiliated with the wedge-algebras $\mathcal{A}_{0}(\mathcal{W})$ whenever supp $f \subset \mathcal{W}$. Since the operators $\phi_{0}(f)$ also generate single particle states from the vacuum, they are polarization free generators in the sense defined above.

We mention as an aside that the dense set of vectors $\mathcal{A}(\mathcal{W})^{\prime} \Omega$ is a core for $\phi_{0}(f)$ for any wedge $\mathcal{W} \supset \operatorname{supp} f$. This implies that, by the preceding general

construction, one would recover $\phi_{0}(f)$ from the single particle state $\phi_{0}(f) \Omega$ and the net.

The full domains of the locally smeared free fields $\phi_{0}(f)$ are not invariant under spacetime translations, but they contain the common core $\mathcal{D}_{0}$ which has this property. As a matter of fact, $\mathcal{D}_{0}$ is also invariant under Lorentz transformations and the vector-valued functions

$$
(\Lambda, x) \rightarrow \phi_{0}(f) U_{0}(\Lambda, x) \Psi
$$

where $U_{0}$ denotes the underlying unitary representation of the Poincaré group, are strongly continuous for each $\Psi \in \mathcal{D}_{0}$. Moreover,

$$
\left\|\phi_{0}(f) U_{0}(\Lambda, x) \Psi\right\| \leq \mathrm{const}
$$

uniformly for all Poincaré transformations $(\Lambda, x)$.

We emphasize that the existence of such a domain $\mathcal{D}_{0}$ on which polarizationfree generators exhibit a "temperate behaviour" with respect to spacetime transformations does not follow from the general theorem. But it seems to be an indispensible requirement if one wants to use these operators in the analysis of collision states and of scattering amplitudes. For that analysis is based on Fourier transformation, which is only meaningful if the underlying functions do not increase too rapidly at infinity. We therefore take a closer look at such temperate generators in the subsequent section.

\section{Temperateness and absence of interaction}

In view of the preceding considerations, we restrict attention now to those theories which admit polarization-free generators with a temperate behaviour with respect to translations.

Definition: A polarization-free generator $G$ is said to be temperate if there is a dense subspace $\mathcal{D}$ of its domain which is stable under translations, such that for any $\Psi \in \mathcal{D}$ the function $x \rightarrow G U(x) \Psi, \Psi \in \mathcal{D}$, is strongly continuous and polynomially bounded in norm for large $x$, and the same holds true also for its adjoint $G^{*}$. The respective subspaces are called domains of temperateness.

It turns out that this regularity requirement imposes severe constraints on the underlying theory and excludes interaction if the dimension of spacetime is larger than two. In the proof of this statement, we restrict attention to massive theories, describing a single scalar particle of mass $m$, so the spectrum of $U$ has the form

$$
\operatorname{sp} U=\{0\} \cup\left\{p \in \mathbb{R}^{d}: p_{0}=\left(\boldsymbol{p}^{2}+m^{2}\right)^{1 / 2}\right\} \cup\left\{p \in \mathbb{R}^{d}: p_{0} \geq\left(\boldsymbol{p}^{2}+4 m^{2}\right)^{1 / 2}\right\},
$$


where $m>0$, but our arguments also apply to theories with a more complex particle spectrum. In a first step we show that temperate polarization-free generators lead to solutions of the Klein-Gordon equation and have in their domains single particle states with compact energy-momentum support about any given point on the "mass shell" $\left\{p \in \mathbb{R}^{d}: p_{0}=\left(\boldsymbol{p}^{2}+m^{2}\right)^{1 / 2}\right\}$.

Lemma 3.1 Let $G$ be a temperate polarization-free generator of mass $m$. Then

(a) $x \rightarrow G(x)=U(x) G U(x)^{-1}$ is a weak solution of the Klein-Gordon equation of mass $m$ on the domain of temperateness $\mathcal{D}$.

(b) The domain $\mathcal{D}$ contains a dense set of vectors with compact spectral support. In particular, there exist single particle states of mass $m$ in $\mathcal{D}$ with spectral support in any given neighborhood of any point on the mass shell.

Corresponding statements hold also for the adjoint $G^{*}$ of $G$.

Proof: (a) If $G$ is affiliated with the wedge algebra $\mathcal{A}(\mathcal{W})$, say, the operators $G(x)$ are affiliated with $\mathcal{A}(\mathcal{W}+x)$ by covariance. Now for $x$ varying in some open, bounded region $\mathcal{U} \subset \mathbb{R}^{d}$, there is a wedge $\mathcal{W}_{0} \supset \cup_{x \in \mathcal{U}}(\mathcal{W}+x)$, hence the operators $G(x)^{*}, x \in \mathcal{U}$, contain the common dense subspace $\mathcal{A}\left(\mathcal{W}_{0}\right)^{\prime} \Omega$ in their domains. Thus for $\Psi \in \mathcal{D}$

$$
\left(G(x) \Psi, A^{\prime} \Omega\right)=\left(\Psi, G(x)^{*} A^{\prime} \Omega\right)=\left(\Psi, A^{\prime} G(x)^{*} \Omega\right), \quad A^{\prime} \in \mathcal{A}\left(\mathcal{W}_{0}\right)^{\prime} .
$$

Since $G^{*} \Omega \in E_{m} \mathcal{H}$, the function $x \rightarrow G(x)^{*} \Omega=U(x) G^{*} \Omega$ is a weak solution of the Klein-Gordon equation, so one obtains from the preceding equation for any test function $f$ with support in $\mathcal{U}$

$$
\int d x\left(\left(\square+m^{2}\right) f^{*}\right)(x)\left(G(x) \Psi, A^{\prime} \Omega\right)=0, \quad A^{\prime} \in \mathcal{A}\left(\mathcal{W}_{0}\right)^{\prime},
$$

where $f^{*}$ is the complex conjugate of $f$. Making use of the temperateness assumption and the Reeh-Schlieder property of $\Omega$, this implies

$$
\int d x\left(\left(\square+m^{2}\right) f\right)(x) G(x) \Psi=0,
$$

where the integral is defined in the strong sense. Since $\mathcal{U}$ was arbitrary, the latter equation extends to all test functions $f \in \mathcal{S}\left(\mathbb{R}^{d}\right)$ by continuity.

(b) The set of vectors of the form $\Psi(f)=\int d x f(x) U(x) \Psi$, where $\Psi \in \mathcal{D}$ and $f$ is any test function whose Fourier transform $\tilde{f}$ has compact support, has compact spectral support and it is dense in $\mathcal{H}$ since $\mathcal{D}$ is dense and $U$ is continuous. By choosing the support of $\widetilde{f}$ properly, one obtains single particle states with spectral support in any given neighborhood of any point on the mass shell. It remains to be shown that these vectors belong to the domain of temperateness of $G$. There holds for any vector $\Phi^{*}$ in the domain of $G^{*}$

$$
\begin{gathered}
\left|\left(\Psi(f), G^{*} \Phi^{*}\right)\right|=\left|\int d x f^{*}(x)\left(U(x) \Psi, G^{*} \Phi^{*}\right)\right| \leq \int d x|f(x)|\left|\left(G U(x) \Psi, \Phi^{*}\right)\right| \\
\leq \int d x|f(x)||| G U(x) \Psi\|\| \Phi^{*}\left\|\leq \int d x|f(x)| Q(x)\right\| \Phi^{*} \|
\end{gathered}
$$


for some polynomial $Q$, depending only on $\Psi$ by the temperateness assumption. Hence $\Psi(f)$ is an element of the domain of $G^{* *}=G$, and the same holds true for $U(x) \Psi(f)=\Psi\left(f_{x}\right)$, where $f_{x}(y)=f(y-x), y \in \mathbb{R}^{d}$, is the translated test function. The continuity of $x \rightarrow G U(x) \Psi(f)$ and temperateness follow from the estimate $\|G(U(x) \Psi(f)-\Psi(f))\| \leq \int d y\left|f_{x}(y)-f(y)\right| Q(y)$ with the same polynomial $Q$ as above. Hence $\Psi(f)$ is an element of the domain of temperateness $\mathcal{D}$. The corresponding statements for $G^{*}$ are established in the same manner.

Picking any single particle state $\Psi_{1} \in \mathcal{D}$ with spectral support in a given compact region on the mass shell, let us turn next to the interpretation of the vectors $G(x) \Psi_{1}$. As $x \rightarrow G(x)$ is a solution of the Klein-Gordon equation, one may expect - guided by the LSZ asymptotic condition - that these vectors describe asymptotic two-particle states. But in view of the weak localization properties of the generators $G$ and the domain problems involved, some care is needed in the analysis.

We rely in our argument on an approach to collision theory established by Hepp [11] for the proof of the LSZ reduction formulas in the general framework of local quantum field theory, cf. also [12]. The main ingredient are quasilocal operators $A\left(f_{t}\right)$ of the form

$$
A\left(f_{t}\right)=\int d x f_{t}(x) A(x) .
$$

Here $A \in \mathcal{A}(\mathcal{O})$ are local operators, where the localization region $\mathcal{O}$ is held fixed in the following, and the functions $f_{t}, t \in \mathbb{R}$, are given by

$$
f_{t}(x)=(2 \pi)^{-d / 2} \int d p \widetilde{f}(p) e^{i\left(p_{0}-\omega_{p}\right) t} e^{-i p x}
$$

where $\tilde{f} \in \mathcal{S}\left(\mathbb{R}^{d}\right)$ and $\omega_{p}=\left(\boldsymbol{p}^{2}+m^{2}\right)^{1 / 2}$. If $\widetilde{f}$ has support in a sufficiently small neighborhood of some point on the mass shell, $A\left(f_{t}\right) \Omega$ is an element of $E_{m} \mathcal{H}$ which does not depend on $t$. Moreover,

$$
\lim _{t \rightarrow \mp \infty} A\left(f_{t}\right) \Phi=A(f)_{\text {out }} \Phi, \quad \lim _{t \rightarrow \mp \infty} A\left(f_{t}\right)^{*} \Phi=A(f)_{\text {out }}^{\text {out }}{ }^{*} \Phi,
$$

where $A(f)_{\text {in }}, A(f)_{\text {out }}$ are the creation operators of an incoming, respectively outgoing particle which is in the state $A(f) \Omega$, and their adjoints $A(f)_{\text {in }}{ }^{*}, A(f)_{\text {out }}{ }^{*}$ are the corresponding annihilation operators.

These asymptotic relations have been established in [11, 12] for some dense set of "decent" collision states $\Phi$. But, making use of the fact that the operator norms $\left\|A\left(f_{t}\right) E(\Delta)\right\|$ and $\left\|A\left(f_{t}\right)^{*} E(\Delta)\right\|$ are uniformly bounded in $t$ for any compact subset $\Delta$ of the spectrum of $U$ [13], they can be extended by continuity to all states with compact spectral support. Thus there holds in particular for the single particle states $\Psi_{1}$ considered above

$$
\lim _{t \rightarrow \mp \infty} A\left(f_{t}\right) \Psi_{1}=\left(A(f) \Omega \times \Psi_{1}\right)_{\text {in }}^{\text {out }}, \quad \lim _{t \rightarrow \mp \infty} A\left(f_{t}\right)^{*} \Psi_{1}=\left(A(f) \Omega, \Psi_{1}\right) \Omega,
$$

where we employ the standard notation for collision states. 
In the subsequent discussion, we will make use of the support properties of the functions $f_{t}$ for asymptotic $t$ [11]. Let

$$
\Gamma(f)=\left\{\left(1, \boldsymbol{p} / \omega_{p}\right): p \in \operatorname{supp} \tilde{f}\right\}
$$

be the "velocity support" of $f$ and let $\chi$ be any smooth function which is equal to 1 on $\Gamma(f)$ and vanishes in the complement of some slightly larger region $\Gamma_{\varepsilon}(f)$. The asymptotically dominant part of $f_{t}$ is given by $x \rightarrow \hat{f}_{t}(x)=\chi(x / t) f_{t}(x)$. It is thus a test function with support in $t \Gamma_{\varepsilon}(f), t \neq 0$. The resulting remainder $x \rightarrow \check{f}_{t}(x)=(1-\chi(x / t)) f_{t}(x)$ tends to zero in the topology of $\mathcal{S}\left(\mathbb{R}^{d}\right)$ as $t \rightarrow \pm \infty$. The decomposition $f_{t}=\hat{f}_{t}+\check{f}_{t}$ will be repeatedly used in the following arguments.

Assuming for the sake of concreteness that the given generator $G$ is affiliated with $\mathcal{A}\left(\mathcal{W}_{1}\right)$, where $\mathcal{W}_{1}$ is the wedge defined in (2.2), we introduce the following partial ordering of sets with reference to that wedge.

Definition: Let $\Gamma_{a}, \Gamma_{b} \subset \mathbb{R}^{d}$ be compact sets. $\Gamma_{a}$ is said to be a precursor of $\Gamma_{b}$, $\Gamma_{a} \prec \Gamma_{b}$ in formula form, if $\Gamma_{b}-\Gamma_{a}$ (the set of all difference vectors) is contained in $\mathcal{W}_{1}$.

Since $\Gamma_{a}, \Gamma_{b}$ are compact, the set $\Gamma_{b}-\Gamma_{a}$ is compact as well. Hence, as $\mathcal{W}_{1}$ is an open cone, it follows from $\Gamma_{a} \prec \Gamma_{b}$ that there is some $\delta>0$ such that $t \Gamma_{b}-t \Gamma_{a} \subset \mathcal{W}_{1}+(0, t \delta, 0, \ldots 0)$ for $t>0$. In view of $\left\{\mathcal{W}_{1}+x\right\}^{\prime}=-\mathcal{W}_{1}+x$, this implies $t \Gamma_{a}+(0, t \delta, 0, \ldots 0) \subset\left(\mathcal{W}_{1}+t \Gamma_{b}\right)^{\prime}, t>0$, i.e. the sets $t \Gamma_{a}$ and $\left(\mathcal{W}_{1}+t \Gamma_{b}\right)$ are spacelike separated and their spatial distance increases linearly with $t$.

We shall apply the above order relation to the velocity supports of test functions, defined in (3.10), as well as to the velocity supports of single particle states, which are defined in an analogous manner by

$$
\Gamma\left(\Psi_{1}\right)=\left\{\left(1, \boldsymbol{p} / \omega_{p}\right): p \in \operatorname{supp} \Psi_{1}\right\}
$$

where $\operatorname{supp} \Psi_{1}$ is the spectral support of $\Psi_{1}$. After these preparations, we can clarify now the interpretation of the vectors $G(g) \Psi_{1}=\int d x g(x) G(x) \Psi_{1}$ in terms of asymptotic two-particle states.

Lemma 3.2 Let $G$ be a temperate polarization-free generator of mass $m$ which is localized in the wedge $\mathcal{W}_{1}$, let $\Psi_{1}$ be a single particle state in its domain $\mathcal{D}$ of temperateness with compact spectral support, and let $g \in \mathcal{S}\left(\mathbb{R}^{d}\right)$ be any test function whose Fourier transform has support in a sufficiently small neighborhood of some point on the mass shell. Then

(a) $G(g) \Psi_{1}=\left(G(g) \Omega \times \Psi_{1}\right)_{\text {in }}$ if $\Gamma(g) \prec \Gamma\left(\Psi_{1}\right)$,

(b) $G(g) \Psi_{1}=\left(G(g) \Omega \times \Psi_{1}\right)_{\text {out }}$ if $\Gamma\left(\Psi_{1}\right) \prec \Gamma(g)$.

Proof: Let $\Psi^{*}$ be any vector in the domain of temperateness of $G^{*}$ with compact spectral support. Then

$$
\left(G(g) \Psi_{1}, \Psi^{*}\right)=\int d x g^{*}(x)\left(\Psi_{1}, G^{*}(x) \Psi^{*}\right)=\left(\Psi_{1}, G^{*}\left(g^{*}\right) \Psi^{*}\right)
$$


where $G^{*}\left(g^{*}\right) \Psi^{*}=\int d x g^{*}(x) G^{*}(x) \Psi^{*}$ is defined as a strong integral. Now by the Reeh-Schlieder property of $\Omega$, there is for any $\delta>0$ an $A \in \mathcal{A}(\mathcal{O})$ and a test function $f$ whose Fourier transform has support in any given neighbourhood of $\operatorname{supp} \Psi_{1}$ such that $\left\|\Psi_{1}-A(f) \Omega\right\|<\delta$ and $A(f) \Omega \in E_{m} \mathcal{H}$. In view of the latter fact, one may replace $f$ in $A(f) \Omega$ by any member of the corresponding family of test functions $f_{t}$, defined in (3.7). Proceeding to the decomposition $f_{t}=\hat{f}_{t}+\check{f}_{t}$ and taking into account that $\left\|A\left(\check{f}_{t}\right)\right\| \leq \int d x\left|\check{f}_{t}(x)\right|\|A\| \rightarrow 0$ as $t \rightarrow \pm \infty$, it follows that

$$
A(f) \Omega=A\left(f_{t}\right) \Omega=\lim _{t \rightarrow \pm \infty} A\left(\hat{f}_{t}\right) \Omega .
$$

Similarly, since $x \rightarrow G^{*}(x) \Psi^{*}$ is a weak solution of the Klein-Gordon equation according to Lemma 3.1, one may replace $g$ in $G^{*}\left(g^{*}\right) \Psi^{*}$ by $g_{t}$. For

$$
\left(g_{t}-g\right)(x)=\left(\square+m^{2}\right)(2 \pi)^{-d / 2} \int d p \frac{\widetilde{g}(p)}{\left(p_{0}+\omega_{p}\right)} \frac{\left(1-e^{i\left(p_{0}-\omega_{p}\right) t}\right)}{\left(p_{0}-\omega_{p}\right)} e^{-i p x},
$$

and the expression under the integral is a test function because of the support properties of $\widetilde{g}$. Making use of the decomposition $g_{t}=\hat{g}_{t}+\check{g}_{t}$ and temperateness, which implies

$$
\left\|G^{*}\left(\check{g}_{t}^{*}\right) \Psi^{*}\right\| \leq \int d x\left|\check{g}_{t}(x)\right|\left\|G^{*} U(x) \Psi^{*}\right\| \leq \int d x\left|\check{g}_{t}(x)\right| Q(x)
$$

for some polynomial $Q$, one finds that $G^{*}\left(\check{g}_{t}^{*}\right) \Psi^{*} \rightarrow 0$ as $t \rightarrow \pm \infty$ and

$$
G^{*}\left(g^{*}\right) \Psi^{*}=G^{*}\left(g_{t}^{*}\right) \Psi^{*}=\lim _{t \rightarrow \pm \infty} G^{*}\left(\hat{g}_{t}^{*}\right) \Psi^{*}
$$

strongly. Combining these facts, one gets

$$
\begin{aligned}
\left(A(f) \Omega, G^{*}\left(g^{*}\right) \Psi^{*}\right) & =\lim _{t \rightarrow \pm \infty}\left(A\left(\hat{f}_{t}\right) \Omega, G^{*}\left(\hat{g}_{t}^{*}\right) \Psi^{*}\right) \\
& =\lim _{t \rightarrow \pm \infty} \int d x \hat{g}_{t}^{*}(x)\left(A\left(\hat{f}_{t}\right) \Omega, G^{*}(x) \Psi^{*}\right)
\end{aligned}
$$

According to the choice of the test function $f$, its velocity support $\Gamma(f)$ is contained in a small neighborhood $\Gamma_{\varepsilon}\left(\Psi_{1}\right)$ of $\Gamma\left(\Psi_{1}\right)$, and consequently the operators $A\left(\hat{f}_{t}\right)$ are localized in $\mathcal{O}+t \Gamma_{\varepsilon}\left(\Psi_{1}\right)$. On the other hand, the operators $G^{*}(x)$, appearing under the integral in (3.17), are affiliated with $\mathcal{A}\left(\mathcal{W}_{1}+x\right)$, $x \in t \Gamma_{\varepsilon}(g)$. Because of locality, they commute with $A\left(\hat{f}_{t}\right)$ on their respective domains if $\mathcal{W}_{1}+t \Gamma_{\varepsilon}(g)$ is spacelike separated from $\mathcal{O}+t \Gamma_{\varepsilon}\left(\Psi_{1}\right)$.

In case (a) of the statement, there holds $-\Gamma\left(\Psi_{1}\right) \prec-\Gamma(g)$ and therefore also $-\Gamma_{\varepsilon}\left(\Psi_{1}\right) \prec-\Gamma_{\varepsilon}(g)$ if the respective neighborhoods are suitably chosen. Hence, according to the above geometrical considerations, the regions $-|t| \Gamma_{\varepsilon}\left(\Psi_{1}\right)$ and $\mathcal{W}_{1}-|t| \Gamma_{\varepsilon}(g)$ are spacelike separated, and their spatial distance increases linearly with $|t|$. Because of the latter fact and since $\mathcal{O}$ is bounded, the two regions $\mathcal{O}+t \Gamma_{\varepsilon}\left(\Psi_{1}\right)$ and $t \Gamma_{\varepsilon}(g)$ are spacelike separated if $t<0$ and $|t|$ is sufficiently 
large. One can then reexpress the integral in (3.17) according to

$$
\begin{aligned}
\int d x \hat{g}_{t}^{*}(x)\left(A\left(\hat{f}_{t}\right) \Omega, G^{*}(x) \Psi^{*}\right) & =\int d x \hat{g}_{t}^{*}(x)\left(\Omega, G^{*}(x) A\left(\hat{f}_{t}\right)^{*} \Psi^{*}\right) \\
& =\left(G\left(\hat{g}_{t}\right) \Omega, A\left(\hat{f}_{t}\right)^{*} \Psi^{*}\right) .
\end{aligned}
$$

In the latter expression, one can reverse now the passage from $f_{t}, g_{t}$ to their respective asymptotically dominant parts, taking into account that, in the limit of asymptotic $t,\left\|G\left(\check{g}_{t}\right) \Omega\right\| \rightarrow 0,\left\|A\left(\check{f}_{t}\right)\right\| \rightarrow 0$, and $\left\|A\left(f_{t}\right)^{*} \Psi^{*}\right\| \leq$ const since $\Psi^{*}$ has compact spectral support [13]. Hence, by a straightforward estimate, one finds that $\left(G\left(\hat{g}_{t}\right) \Omega, A\left(\hat{f}_{t}\right)^{*} \Psi^{*}\right)$ and $\left(G\left(g_{t}\right) \Omega, A\left(f_{t}\right)^{*} \Psi^{*}\right)=\left(A\left(f_{t}\right) G(g) \Omega, \Psi^{*}\right)$ have the same limit as $t \rightarrow-\infty$. Plugging this information into relation (3.17) and making use of the asymptotic formula (3.9), one arrives at

$$
\begin{aligned}
\left(A(f) \Omega, G^{*}\left(g^{*}\right) \Psi^{*}\right) & =\lim _{t \rightarrow-\infty}\left(A\left(f_{t}\right) G(g) \Omega, \Psi^{*}\right) \\
& =\left((A(f) \Omega \times G(g) \Omega)_{i n}, \Psi^{*}\right) .
\end{aligned}
$$

In the resulting equation, one can replace $A(f) \Omega$ by $\Psi_{1}$ since $\left\|\Psi_{1}-A(f) \Omega\right\|<\delta$, where $\delta>0$ was arbitrary, and

$$
\left\|\left(\Psi_{1} \times G(g) \Omega\right)_{i n}-(A(f) \Omega \times G(g) \Omega)_{i n}\right\| \leq \sqrt{2}\left\|\Psi_{1}-A(f) \Omega\right\|\|G(g) \Omega\|,
$$

by the Fock structure of collision states. In view of relation (3.12), this completes the proof of part (a) of the statement. The proof of part (b) is similar, but now one has to take into account that the regions $\mathcal{O}+t \Gamma_{\varepsilon}\left(\Psi_{1}\right)$ and $t \Gamma_{\varepsilon}(g)$ are spacelike separated if $t>0$ is sufficiently large. So in this case one arrives at an interpretation of the vectors $G(g) \Psi_{1}$ in terms of outgoing collision states.

In the next step, we establish a weak form of commutation relations between the operators $G(x)$ and the asymptotic creation and annihilation operators. The result will enable us to compute scattering amplitudes.

Lemma 3.3 Let $\Psi, \Psi^{*}$ be vectors with compact spectral support in the domains of temperateness of $G$ and $G^{*}$, respectively, and let $f, g$ be test functions whose Fourier transforms have support in small neighborhoods of points on the mass shell. Then

(a) $\left(G(g) \Psi, A(f)_{\text {in }}^{\bullet} \Psi^{*}\right)=\left(A(f)_{\text {in }}^{\bullet *} \Psi, G^{*}\left(g^{*}\right) \Psi^{*}\right) \quad$ if $\quad \Gamma(g) \prec \Gamma(f)$,

(b) $\left(G(g) \Psi, A(f)_{\text {out }} \bullet^{*}\right)=\left(A(f)_{\text {out }}{ }^{*} \Psi, G^{*}\left(g^{*}\right) \Psi^{*}\right) \quad$ if $\quad \Gamma(f) \prec \Gamma(g)$.

Here the symbol $X^{\bullet}$ stands for both, the operator $X$ and its adjoint $X^{*}$.

Proof: The argument is very similar to the proof of the preceding lemma and it therefore suffices to indicate the main steps. In case (a) one has, in view of the fact that $G\left(g_{t}\right) \Psi=G(g) \Psi, t \in \mathbb{R}$, and the asymptotic relation (3.8),

$$
\begin{aligned}
\left(G(g) \Psi, A(f)_{i n}^{\bullet} \Psi^{*}\right) & =\left(G\left(g_{t}\right) \Psi, A(f)_{i n}^{\bullet} \Psi^{*}\right) \\
=\lim _{t \rightarrow-\infty}\left(G\left(g_{t}\right) \Psi, A\left(f_{t}\right)^{\bullet} \Psi^{*}\right) & =\lim _{t \rightarrow-\infty}\left(G\left(\hat{g}_{t}\right) \Psi, A\left(\hat{f}_{t}\right)^{\bullet} \Psi^{*}\right),
\end{aligned}
$$


where, in the last step, $f_{t}, g_{t}$ have been replaced by their asymptotically dominant parts. Since $\Gamma(g) \prec \Gamma(g)$, the regions $\mathcal{W}_{1}+t \Gamma_{\varepsilon}(g)$ and $\mathcal{O}+t \Gamma_{\varepsilon}(f)$ are spacelike separated for $t<0$ and $|t|$ sufficiently large. Hence, by locality,

$$
\begin{aligned}
\lim _{t \rightarrow-\infty}\left(G\left(\hat{g}_{t}\right) \Psi, A\left(\hat{f}_{t}\right)^{\bullet} \Psi^{*}\right) & =\lim _{t \rightarrow-\infty}\left(A\left(\hat{f}_{t}\right)^{\bullet *} \Psi, G^{*}\left(\hat{g}_{t}^{*}\right) \Psi^{*}\right) \\
=\lim _{t \rightarrow-\infty}\left(A\left(f_{t}\right)^{\bullet *} \Psi, G^{*}\left(g_{t}^{*}\right) \Psi^{*}\right) & =\left(A(f)_{i n}^{\bullet *} \Psi, G^{*}\left(g^{*}\right) \Psi^{*}\right),
\end{aligned}
$$

where, in the second equality, the transition from $f_{t}, g_{t}$ to the asymptotically dominant parts has been reversed and, in the last step, the asymptotic relation (3.8) has been used as well as the fact that $G^{*}\left(g_{t}{ }^{*}\right) \Psi^{*}=G^{*}\left(g^{*}\right) \Psi^{*}, t \in \mathbb{R}$. This establishes statement (a). The proof of (b) is analogous.

In a final step, we have to determine the spectral support of $G \Omega$ with respect to the spatial momentum operators $\boldsymbol{P}$ in order to see which single particle states can be generated by $G$ from the vacuum.

Lemma 3.4 Let $G$ be a polarization-free generator which is affiliated with the algebra $\mathcal{A}\left(\mathcal{W}_{1}\right)$. The spectral support of $G \Omega$ with respect to the spatial momentum operators $\boldsymbol{P}=\left(P_{1}, P_{2}, \ldots P_{d-1}\right)$ is equal to $\mathbb{R} \times \mathcal{C}$, where $\mathcal{C} \subset \mathbb{R}^{d-2}$ is a closed set with open interior.

Proof: Let $A$ be any local operator which is localized in $\mathcal{W}_{1}^{\prime}$. Since $x \rightarrow G(x) \Omega$ and $x \rightarrow G^{*}(x) \Omega$ are solutions of the Klein-Gordon equation of mass $m$, the commutator function

$$
x \rightarrow C(x)=(A \Omega, G(x) \Omega)-\left(G^{*}(x) \Omega, A^{*} \Omega\right)
$$

can be represented in the form

$$
C(x)=\int \frac{d \boldsymbol{p}}{2 \omega_{p}}\left(K_{+}(\boldsymbol{p}) e^{i \omega_{p} x_{0}}-K_{-}(\boldsymbol{p}) e^{-i \omega_{p} x_{0}}\right) e^{-i \boldsymbol{p} \boldsymbol{x}} .
$$

Here the functions $K_{ \pm}$are given by

$$
\begin{aligned}
& K_{+}(\boldsymbol{p})=(A \Omega)(\boldsymbol{p})^{*}(G \Omega)(\boldsymbol{p}), \\
& K_{-}(\boldsymbol{p})=\left(G^{*} \Omega\right)(-\boldsymbol{p})^{*}\left(A^{*} \Omega\right)(-\boldsymbol{p}),
\end{aligned}
$$

where $\boldsymbol{p} \rightarrow(A \Omega)(\boldsymbol{p})$ is the momentum space wave function of the single particle vector $E_{m} A \Omega$, and similarly for the other terms.

Because of the localization properties of the operators $A$ and $G$, the commutator function $x \rightarrow C(x)$ and its time derivative vanish at time $x_{0}=0$ in the half space $\left\{\boldsymbol{x} \in \mathbb{R}^{d-1}: x_{1}>0\right\}$. In view of the representation (3.24), this implies that the functions

$$
\boldsymbol{p} \rightarrow \frac{1}{\omega_{p}}\left(K_{+}(\boldsymbol{p})-K_{-}(\boldsymbol{p})\right) \quad \text { and } \quad \boldsymbol{p} \rightarrow\left(K_{+}(\boldsymbol{p})+K_{-}(\boldsymbol{p})\right)
$$

can be analytically continued in $p_{1}$ into the lower half plane. As $\boldsymbol{p} \rightarrow \omega_{p}$ is analytic in $p_{1}$ in a strip about the origin, the functions $\boldsymbol{p} \rightarrow K_{ \pm}(\boldsymbol{p})$ can likewise be analytically continued in $p_{1}$ into some strip of the lower half plane. 
Now if $\mathcal{U} \subset \mathbb{R}$ and $\mathcal{V} \subset \mathbb{R}^{d-2}$ are open sets such that $\boldsymbol{p} \rightarrow(G \Omega)(\boldsymbol{p})$ vanishes for almost all spatial momenta $\boldsymbol{p}=\left(p_{1}, \boldsymbol{p}_{\perp}\right)$ with $p_{1} \in \mathcal{U}$ and $\boldsymbol{p}_{\perp} \in \mathcal{V}$, the function $\boldsymbol{p} \rightarrow K_{+}(\boldsymbol{p})$ vanishes for these momenta as well. Being the boundary value of an analytic function with respect to $p_{1}, K_{+}(\boldsymbol{p})$ therefore vanishes for all $p_{1} \in \mathbb{R}$ and $\boldsymbol{p}_{\perp} \in \mathcal{V}$. Since $A \in \mathcal{A}\left(\mathcal{W}_{1}{ }^{\prime}\right)$ was arbitrary and the set of single particle states $E_{m} A \Omega, A \in \mathcal{A}\left(\mathcal{W}_{1}{ }^{\prime}\right)$, is dense in $E_{m} \mathcal{H}$, this implies $(G \Omega)(\boldsymbol{p})=0$ for $p_{1} \in \mathbb{R}$ and $\boldsymbol{p}_{\perp} \in \mathcal{V}$. Thus the complement of the support of $\boldsymbol{p} \rightarrow(G \Omega)(\boldsymbol{p})$ in momentum space $\mathbb{R}^{d-1}$ has the form $\mathbb{R} \times \mathcal{V}$, where $\mathcal{V} \subset \mathbb{R}^{d-2}$ is open. Hence, disregarding sets of measure 0 , the statement follows since $G \Omega$ is different from zero.

We have accumulated now sufficient information in order to proceed to the computation of the scattering amplitudes in the underlying theory, provided the dimension of spacetime is larger than two. Let $\boldsymbol{p}=\left(p_{1}, \boldsymbol{p}_{\perp}\right)$ be any vector in the spectral support of $G \Omega$ with respect to the spatial momentum operators and let $p_{1}<0$ and $\boldsymbol{p}_{\perp} \neq 0$. We pick a test function $g$ whose Fourier transform has support in a sufficiently small neighborhood of $\left(\omega_{p}, \boldsymbol{p}\right)$, and a single particle state $\Psi_{1}$ which is an element of the domain of temperateness of $G$ with spectral support in a small neighborhood of $\left(\omega_{p},-\boldsymbol{p}\right)$. Hence $\Gamma(g) \prec \Gamma\left(\Psi_{1}\right)$ and consequently $G(g) \Psi_{1}=\left(G(g) \Omega \times \Psi_{1}\right)_{\text {in }}$ by Lemma 3.2 .

As $\boldsymbol{p}_{\perp} \neq 0$, there are spatial momenta $\boldsymbol{q}$ with $q_{1}<p_{1}$ and $|\boldsymbol{q}|=|\boldsymbol{p}|$ (here the dimension of spacetime enters). For any such $\boldsymbol{q}$, we choose a test function $f$ whose Fourier transform has support in a small neighborhood of $\left(\omega_{q}, \boldsymbol{q}\right)$ such that $\Gamma(f) \prec$ $\Gamma(g)$. Finally, we pick a single particle state $\Psi_{1}^{*}$ in the domain of temperateness of $G^{*}$ with spectral support about $\left(\omega_{q},-\boldsymbol{q}\right)$. After these preparations, we can apply Lemma 3.3 and compute

$$
\begin{gathered}
\left(\left(G(g) \Omega \times \Psi_{1}\right)_{\text {in }},\left(A(f) \Omega \times \Psi_{1}^{*}\right)_{\text {out }}\right)=\left(G(g) \Psi_{1}, A(f)_{\text {out }} \Psi_{1}^{*}\right) \\
=\left(A(f)_{\text {out }}^{*} \Psi_{1}, G^{*}\left(g^{*}\right) \Psi_{1}^{*}\right)=0,
\end{gathered}
$$

where, in the last step, we used the fact that $A(f)$ out ${ }^{*} \Psi_{1}=\left(A(f) \Omega, \Psi_{1}\right) \Omega=0$ since $\Gamma(f) \prec \Gamma\left(\Psi_{1}\right)$. Varying $f, g$ and $\Psi_{1}, \Psi_{1}^{*}$ within the above limitations, it follows that elastic scattering processes of two particles with initial momenta about $\boldsymbol{p},-\boldsymbol{p}$ and final momenta about $\boldsymbol{q},-\boldsymbol{q}-$ although admitted by the energymomentum conservation law - do not occur in the underlying theory.

This result implies that the elastic two-particle scattering amplitude $T$ vanishes identically. For the proof of this statement, we recall that in a relativistic theory $T=T(s, t)$ is a distribution with respect to the invariants $s, t$ (the squares of the energy in the center of mass system and the momentum transfer, respectively). Thus for $\boldsymbol{p}, \boldsymbol{q}$ as above, $s=4\left(m^{2}+\boldsymbol{p}^{2}\right)$ and $t=-2 \boldsymbol{p}^{2}(1-\cos \theta)$, where $\theta$ is the scattering angle. It is a well-known consequence of locality, relativistic covariance, and the form of the energy-momentum spectrum that $T(s, t)$ is, in the physical region $s \geq 4 m^{2}-t$ for fixed $t \leq 0$, the boundary value from $\operatorname{Im} s>0$ of an analytic function in the cut $s$-plane. On the other hand, for fixed $s$, it is analytic in the variable $\cos (\theta)$ in the Lehmann ellipse [14] with foci at \pm 1 and semi-minor axis of length $6 m^{2} / \sqrt{s\left(s-4 m^{2}\right)}$, cf. 115 for an exposition of these basic facts. 
By the preceding computations, we know that the scattering amplitude vanishes for $s, t$ in some open set. In fact, taking into account that all momenta

$\boldsymbol{p}=\left(p_{1}, \boldsymbol{p}_{\perp}\right)$ with $p_{1} \in \mathbb{R}_{-}$belong to the spectral support of $G \Omega$ for some fixed $\boldsymbol{p}_{\perp} \neq 0$ (cf. Lemma 3.4) and varying $\boldsymbol{q}$ within the above limitations, we get

$$
T(s, t)=0 \quad \text { for } \quad s>4\left(m^{2}+\left|\boldsymbol{p}_{\perp}\right|^{2}\right), \quad 0>t>-4\left|\boldsymbol{p}_{\perp}\right|^{2} .
$$

By analyticity in $\cos (\theta)$, this equality extends to all scattering angles and hence to all $t$ for the given range of $s$. Analyticity of $T(s, t)$ in $s$ then implies that the scattering amplitude vanishes everywhere. It is a well-known consequence of this result that then there can be no non-trivial multi particle scattering or particle production either [16]. These implications hold in any number $d>2$ of spacetime dimensions [17]. So we arrive at the following statement.

Theorem 3.5 If in a local, relativistic quantum field theory of a scalar massive particle in $d>2$ spacetime dimensions there exists a temperate polarization-free generator, then the scattering matrix is trivial.

For the sake of simplicity, we have restricted attention in the preceding analysis to theories describing a single scalar massive particle. But it should be clear from our discussion that similar results hold in theories with a more complex particle spectrum. There one finds that particles whose states can be generated from the vacuum by temperate polarization-free generators do not participate in collision processes. For the derivation of this result it is actually not necessary to assume that the collision states can be constructed by local operators. Cone-like localized "interpolating operators", whose existence has been established in all theories of massive particles [18], are completely sufficient for the proof. We therefore conclude that in the presence of interaction there is no room for temperate polarization-free generators in more than two spacetime dimensions.

\section{Polarization-free generators in two dimensions}

The analysis in the preceding section did not lead to any restrictions on the form of the elastic scattering amplitudes in two spacetime dimensions. For configurations of asymptotic particle momenta which would allow one to show that the scattering amplitudes have to vanish in the presence of temperate polarizationfree generators cannot occur in this case because of the energy-momentum conservation law. So non-trivial theories admitting polarization-free generators can and do exist in two spacetime dimensions 4 . It seems therefore worthwhile to have a closer look at the type of constraints imposed on such theories from the present general point of view.

In order to abbreviate this discussion, we assume in the following that the domains of temperateness of $G$ and $G^{*}$ contain incoming and outgoing collision states for arbitrary configurations of particle momenta. Lemma 3.3 then implies 
that on these states

$$
\begin{array}{lll}
G(g) A(f)_{\text {in }}{ }^{*}=A(f)_{\text {in }}{ }^{*} G(g) & \text { if } & \Gamma(g) \prec \Gamma(f), \\
G(g) A(f)_{\text {out }}=A(f)_{\text {out }} G(g) & \text { if } & \Gamma(f) \prec \Gamma(g),
\end{array}
$$

and similarly for $G^{*}\left(g^{*}\right)$. For the lemma says that on the domain of temperateness $G^{*}\left(g^{*}\right)^{*} A(f)_{i n}{ }^{*} \supset A(f)_{i n}{ }^{*} G(g)$ if $\Gamma(g) \prec \Gamma(f)$, say, and with the above domain assumptions one can replace the triple-star expression $G^{*}\left(g^{*}\right)^{*}$ by $G(g)$.

It turns out that these commutation relations imply that there can be no particle production in the underlying theory. In the proof of this statement, we make use of the following lemma.

Lemma 4.1 Let $f, g_{1}, \ldots g_{n}$ be test functions whose Fourier transforms have support about points on the mass shell such that $\Gamma\left(g_{1}\right) \prec \ldots \prec \Gamma\left(g_{n}\right) \prec \Gamma(f)$ and let $A, A_{1}, \ldots A_{n} \in \mathcal{A}(\mathcal{O})$ be arbitrary local operators. Then

$$
A(f)_{\text {in }}{ }^{*}\left(A_{1}\left(g_{1}\right) \Omega \times \cdots \times A_{n}\left(g_{n}\right) \Omega\right)_{\text {out }}=0 .
$$

Proof: The proof is based on induction in $n$. For $n=1$, one has

$$
A(f)_{\text {in }}{ }^{*} A_{1}\left(g_{1}\right) \Omega=\left(A(f) \Omega, A_{1}\left(g_{1}\right) \Omega\right) \Omega=0
$$

because of the support properties of $f, g_{1}$ in momentum space. Assuming that the statement holds for $n$, let $g$ be a test function whose Fourier transform has support about points on the mass shell such that $\Gamma\left(g_{1}\right) \prec \ldots \prec \Gamma\left(g_{n}\right) \prec \Gamma(g) \prec \Gamma(f)$. It then follows from relation (4.2) that

$$
\begin{gathered}
G(g) A_{1}\left(g_{1}\right)_{\text {out }} \cdots A_{n}\left(g_{n}\right)_{\text {out }} \Omega=A_{1}\left(g_{1}\right)_{\text {out }} \cdots A_{n}\left(g_{n}\right)_{\text {out }} G(g) \Omega \\
=\left(A_{1}\left(g_{1}\right) \Omega \times \cdots \times A_{n}\left(g_{n}\right) \Omega \times G(g) \Omega\right)_{\text {out }} .
\end{gathered}
$$

Hence, by relation (4.1) and the induction hypothesis, one obtains

$$
\begin{aligned}
A(f)_{\text {in }}{ }^{*} & \left(A_{1}\left(g_{1}\right) \Omega \times \cdots \times A_{n}\left(g_{n}\right) \Omega \times G(g) \Omega\right)_{\text {out }} \\
& =A(f)_{\text {in }}{ }^{*} G(g) A_{1}\left(g_{1}\right)_{\text {out }} \cdots A_{n}\left(g_{n}\right)_{\text {out }} \Omega \\
& =G(g) A(f)_{\text {in }}{ }^{*} A_{1}\left(g_{1}\right)_{\text {out }} \cdots A_{n}\left(g_{n}\right)_{\text {out }} \Omega=0 .
\end{aligned}
$$

Now let $A_{n+1} \in \mathcal{A}(\mathcal{O})$ be any local operator and $g_{n+1}$ any test function such that $\Gamma\left(g_{1}\right) \prec \ldots \prec \Gamma\left(g_{n}\right) \prec \Gamma\left(g_{n+1}\right) \prec \Gamma(f)$. There exists for given $\delta>0$ a test function $g$ as in the preceding step such that $\left\|A_{n+1}\left(g_{n+1}\right) \Omega-G(g) \Omega\right\|<\delta$. For the spectral support of $G \Omega$ consists of the whole mass shell according to Lemma 3.4 and consequently the set of vectors $\{G(g) \Omega: \operatorname{supp} \widetilde{g} \subset \Delta\}$ is, for any compact set $\Delta \subset \mathbb{R}^{2}$, dense in the corresponding spectral subspace $E(\Delta) E_{m} \mathcal{H}$ of single particle states. As the collision states are continuous with respect to their single particle components, one can thus replace in equation (4.6) the vector $G(g) \Omega$ by $A_{n+1}\left(g_{n+1}\right) \Omega$, proving the statement. 
Let us consider now an incoming collision state of two particles with momenta $p_{1}, p_{2}$ on the mass shell and an outgoing state of $n>2$ particles with mutually different momenta $q_{1}, \ldots q_{n}$. Taking advantage of the fact that in $d=2$ spacetime dimensions the momenta on the mass shell are linearly ordered, we may assume without loss of generality that $q_{1}<\cdots<q_{n}$. Now if the incoming state is to evolve with non-zero probability into this outgoing state, the energy-momentum conservation law requires that $p_{1}+p_{2}=q_{1}+\cdots+q_{n}$. Taking into account that the linear order of momenta is preserved under proper othochronous Lorentz transformations, it is not difficult to see that at least one of the incoming particle momenta, say $p_{1}$, has to be strictly larger than any one of the outgoing momenta, i.e. $q_{1}<\cdots<q_{n}<p_{1}$. We pick now test functions $f_{1}, f_{2}$ and $g_{1}, \ldots g_{n}$ which, in momentum space, have support about $p_{1}, p_{2}$ and $q_{1}, \ldots q_{n}$, respectively, such that $\Gamma\left(g_{1}\right) \prec \ldots \prec \Gamma\left(g_{n}\right) \prec \Gamma\left(f_{1}\right)$. Thus, for any choice of local operators $A_{1}, \ldots A_{n+2} \in \mathcal{A}(\mathcal{O})$, we obtain with the help of the preceding lemma

$$
\begin{aligned}
& \left(\left(A_{1}\left(f_{1}\right) \Omega \times A_{2}\left(f_{2}\right) \Omega\right)_{\text {in }},\left(A_{3}\left(g_{1}\right) \Omega \times \cdots \times A_{n+2}\left(g_{n}\right) \Omega\right)_{\text {out }}\right) \\
& \quad=\left(A_{1}\left(f_{1}\right)_{\text {in }} A_{2}\left(f_{2}\right) \Omega,\left(A_{3}\left(g_{1}\right) \Omega \times \cdots \times A_{n+2}\left(g_{n}\right) \Omega\right)_{\text {out }}\right) \\
& \quad=\left(A_{2}\left(f_{2}\right) \Omega, A_{1}\left(f_{1}\right)_{\text {in }}{ }^{*}\left(A_{3}\left(g_{1}\right) \Omega \times \cdots \times A_{n+2}\left(g_{n}\right) \Omega\right)_{\text {out }}\right)=0 .
\end{aligned}
$$

But the set of collision states with non-overlapping momenta is dense in the set of all collision states, so we arrive at the conclusion that an incoming collision state of two particles can never evolve into an outgoing collision state containing more than two particles, i.e. there is no particle production in the underlying theory.

Theorem 4.2 If a local, relativistic quantum field theory in $d=2$ spacetime dimensions admits temperate polarization-free generators, there is no particle production.

This result shows that in $d=2$ dimensions temperate polarization-free generators can only exist in the presence of additional conservation laws, besides energy-momentum conservation. We have illustrated this fact on the example of the particle number. By a more refined analysis, one can show that also the individual particle momenta have to be preserved in multi-particle collisions. This brings us close to the structure of scattering amplitudes found in completely integrable models. In particular, the apparently general Ansatz for correlation functions of polarization-free generators, proposed by one of the authors in [1, 19, falls back to this special class of theories. Moreover, there are indications in the present general setting that temperate polarization-free generators necessarily have algebraic properties of the type found in these examples. Thus the notion of temperate polarization-free generator may not only be useful for the characterization of such integrable models, but it might also serve as a tool for their general analysis and classification. This interesting aspect of the present investigation will be discussed elsewhere. 


\section{Concluding remarks}

Harry Lehmann, one of the pioneers of the rigorous approach to relativistic quantum field theory, liked to mock at the sometimes cumbersome subtleties appearing in this setting as "problems of inessential selfadjointness". But his scientific work provides ample evidence to the effect that he was willing to invest mathematical diligence and care where the physical context required it.

In the present article, we have encountered a surprisingly subtle feature of relativistic quantum field theory: Mathematics tells us, on one hand, that any such theory accommodates well-defined polarization-free generators. Physics, on the other hand, implies that these generators necessarily have rather peculiar domain properties which do not allow one to apply methods of Fourier analysis. Their relation to the asymptotic particle interpretation is thereby obscured. Being sloppy with regard to these domain properties, one would be led to the unpleasant conclusion that the fundamental postulates of relativistic quantum field theory exclude interaction in more than two spacetime dimensions. Thus it is this subtle point which provides the loophole for theories with non-trivial interaction.

Temperate polarization-free generators exist, however, in two-dimensional integrable models and the present results indicate that they are a distinctive feature of such theories. This fact may be attributed to the presence of large groups of conservation laws in such theories which help to restrain the polarization effects of local operations. We believe that a more detailed investigation of these temperate generators is warranted and will lead to a better understanding of the specific features of these models.

There is another aspect of the present analysis which deserves mentioning, namely the problem of particle statistics in low dimensions. We have discussed here only the simple case of bosons, the case of fermions being similar. But it is well-known that particles in two and three spacetime dimensions can also have anyonic or plektonic statistics (cf. [20, 21] for a systematic analysis of this issue). There is also a general collision theory for such particles [22], but it is an open question whether there exists some kind of associated free fields.

A negative result to that effect is due to Mund [23], who proved that there are no operator-valued solutions of the Klein-Gordon equation which generate such particles from the vacuum and are localized in salient (pointed) spacelike cones. This ad hoc assumption about the localization is, however, crucial for the proof of this no-go theorem. In fact, as in theories of massive anyons and plektons there are still cone-like localized (vacuum polarizing) operators which generate the states of physical interest from the vacuum, one can establish the existence of wedge-localized polarization-free generators for these particles. Taking temperateness as an additional input, it may well be possible to construct from these generators in a systematic manner examples of anyonic or even plektonic theories which come close to the idea of a free field theory. 


\section{References}

[1] H. Lehmann, K. Symanzik and W. Zimmermann: Zur Formulierung quantisierter Feldtheorien, Nuovo Cimento 1 (1955) 205-225.

[2] M. Karowski and P. Weisz: Exact form-factors in (1+1)-dimensional field theoretic models with soliton behavior, Nucl. Phys. B139 (1978) 455-476.

[3] H. Babujian, A. Fring, M. Karowski and A. Zapletal: Exact form-factors in integrable quantum field theories: the Sine-Gordon model, Nucl. Phys. B538 (1999) $535-586$.

[4] B. Schroer: Modular wedge localization and the $d=(1+1)$ form-factor program, Ann. Phys. (N.Y.) 275 (1999) 190-223.

[5] R. Haag: Local Quantum Physics: Fields, Particles, Algebras, Springer 1996.

[6] H.-J. Borchers and J. Yngvason: From quantum fields to local von Neumann algebras, Rev. Math. Phys. Special issue (1992) 15-47.

[7] M. Takesaki: Tomita's Theory of Modular Hilbert Algebras and its Applications, Lecture Notes in Mathematics, Vol. 128, Springer 1970.

[8] H.-J. Borchers: On revolutionizing quantum field theory with Tomita's modular theory, Preprint http://www.lpq.uni-goettingen.de/papers/99/04/99042900.htm, to appear in Journ. Math. Phys.

[9] J. Bisognano and E.H. Wichmann: On the duality condition for a hermitian scalar field, J. Math. Phys. 16 985-1007 (1975). On the duality condition for quantum fields, J. Math. Phys. 17 303-321 (1976).

[10] R. Jost: The General Theory of Quantized Fields, American Math. Soc. 1965.

[11] K. Hepp: On the connection between Wightman and LSZ quantum field theory, pp. 135-246 in: Brandeis University Summer Institute in Theoretical Physics 1965, "Axiomatic Field Theory", Vol. 1 (M. Chretien and S. Deser eds.), Gordon and Breach 1966.

[12] H. Araki: Mathematical Theory of Quantum Fields, Int. Series of Monographs on Physics 101, Oxford Univ. Press 1999.

[13] D. Buchholz: Harmonic analysis of local operators, Commun. Math. Phys. 129 (1990) 631-641.

[14] H. Lehmann: Analytic properties of scattering amplitudes as functions of momentum transfer, Nuovo Cimento 10 (1958) 579-589.

[15] A. Martin: Scattering Theory: Unitarity, Analyticity and Crossing, Lecture Notes in Physics, Vol. 3, Springer 1969.

[16] S. Åks: Proof that scattering implies production in quantum field theory, Journ. Math. Phys. 6 (1965) 516-532. 
[17] J. Bros: private communication.

[18] D. Buchholz and K. Fredenhagen: Locality and the structure of particle states, Commun. Math. Phys. 84 (1982) 1-54.

[19] B. Schroer: Particle physics and QFT at the turn of the century: Old principles with new concepts. (An essay on local quantum physics.), Preprint hep-th/9810080, to appear in Journ. Math. Phys.

[20] K. Fredenhagen, K.H. Rehren and B. Schroer: Superselection sectors with braid group statistics and exchange algebras. 1. General theory, Commun. Math. Phys. 125 (1989) 201-226.

[21] J. Fröhlich and P.A. Marchetti: Quantum field theories of vortices and anyons, Commun. Math. Phys. 121 (1989) 177-223.

[22] K. Fredenhagen and M.R. Gaberdiel: Scattering states of plektons (particles with braid group statistics) in (2+1)-dimensional quantum field theory, Commun. Math. Phys. 175 (1996) 319-336.

[23] J. Mund: No go theorem for "free" relativistic anyons in $(2+1)$-dimension, Lett. Math. Phys. 43 (1998) 319-328. 